\title{
Features of self-learning physical culture during distance education
}

\author{
Sergey Chalohyan ${ }^{l}$, Svetlana Grigan ${ }^{2 *}$, and Gennady Lyashko ${ }^{2}$ \\ ${ }^{1}$ Don State Technical University, 344002, Rostov-on-Don, Russia \\ ${ }^{2}$ Rostov State Transport University, 344038, Rostov-on-Don, Russia
}

\begin{abstract}
On March 14, 2020, an order was posted on the website of the Ministry of Science and Higher Education of the Russian Federation on new rules for organizing educational activities in Russian universities, heads of higher educational institutions were instructed to organize interaction between students and teachers in the educational process in a distance format. Self-study contributes not only to the development of physical skills and health, but also helps to better assimilate the educational material, since the body must periodically receive a "jolt" between long "periods of sitting" in other disciplines. The topic of self-study is especially relevant during periods of a pandemic, when many educational institutions transfer their students to "distance education" and organized physical education classes become impossible. At this time, everyone should understand for himself that it is necessary to perform the exercises, because in such conditions most of the time is spent sitting, while not being active. The number of movements performed per day becomes too low. Therefore, daily physical activities are indispensable and necessary in everyone's life, an alternative solution to the gym is needed, and the work offers a set of exercises at home for distance learning students.
\end{abstract}

\section{Introduction}

On March 14, two thousand and twentieth, an order was posted on the website of the Ministry of Science and Higher Education of the Russian Federation on new rules for organizing educational activities in Russian universities. In order to prevent the spread of the new Coronavirus infection as much as possible, the heads of higher educational institutions were instructed to organize the interaction of students and teachers in the educational process in a distance format. Such a system came into effect on the sixteenth of March two thousand and twenty. Our university was no exception, and we switched to "self-isolation". Unfortunately, today the situation remains tense, because the organization of educational work is still carried out in a remote format.

In the law "On Education of the Russian Federation" "E-learning is understood as the organization of educational activities using information contained in databases and used in the implementation of educational programs and information technologies, technical means, as well as information and telecommunication networks that provide transmission over the

\footnotetext{
* Corresponding author: svetlana-grigan@mail.ru
} 
lines communication of the specified information, the interaction of students and teaching staff. Distance learning technologies are understood as educational technologies implemented mainly with the use of information and telecommunication networks with indirect (at a distance) interaction between students and teachers. "

In mid-2017, the state program "Digital Economy of the Russian Federation" was adopted. In everyday life, the words "digitize", "digitalization", "digital technologies", "digital transformation" are increasingly used. Forums and conferences on this topic began to be held regularly.

The concept of "digital transformation" is the next stage of development, a whole complex of transformations of the organization itself, including changes in the business model, principles of its work and business culture based on technological innovations and solutions.

Digital transformation is not only economy, but a global phenomenon that affects our entire life. It touches on public administration, sets new business practices, and changes our day-to-day practices.

In our country, social security, education, medicine, industry are much more significant areas of attention, the effect of positive changes in which will be felt much more.

And digital technologies themselves, as a tool for transforming the economy, were noticed by the government only after suddenly, in 2017 , it turned out that the share of the digital economy in Russia's GDP is as much as 3.9\%. This is several times lower than that of the leading countries, for example, the USA, Singapore, Israel, but the volume of this market will grow significantly, according to McKinsey, which released the Digital Russia: New Reality report in July 2017. According to them, digitalization will provide from 19 to $34 \%$ of Russia's GDP growth, and the share of the digital economy itself may amount to 8$10 \%$ of GDP.

Digital transformation in the field of "physical culture and sports" is inevitable. It is the matter of time. But one needs to be ready for it mentally, organizationally and financially. Relative closedness in informational terms, leads to a gradual lag both in terms of commercial and technological. Year by year technologies in conducting physical culture classes are playing an increasing role in education, and staying in isolation in this sphere is fraught with subsequent systematic lag behind world leaders.

Technology itself is neither good nor bad. The question of their competent use as a potentially useful tool comes to the fore. Here we can distinguish two layers of technologies that affect the pedagogical industry: the Internet of Things and the integration of the living and the digital.

In our opinion, one of the possible options is the following:

1. At the state level, revise the Charters of organizations, in which the goals of their existence should be more clearly defined in favor of effective activity, and not sluggish processes;

2. Form a federal structure responsible for the digital transformation of the industry. The framework should analyze the goals and potential possibilities for changes in pedagogical programs.;

3. Develop a strategy and roadmap for digital transformation of the industry.

In connection with the regime of self-isolation of the population in Rostov-on-Don, the number of classes at home should be increased. Since self-isolation implies being in a quarantine mode at home, in this situation, students have limited mobility, respectively, motor tasks for students of different ages are not adequately solved. It is recommended to distribute the load during the week in accordance with the age, level of preparedness and functional abilities of the students. The nature, structure and content of independent physical exercises are offered by the teacher of the physical culture or by the coach, if the student is engaged in the sports section. The duration of classes is determined by the 
teacher or indicated in the recommendations for independent physical exercises.

In the course of organizing physical culture classes, the teacher solves various problems. In the distance learning mode, there are no pedagogical realities for solving all the goals of the lesson (educational, health-improving, educational) during direct interaction with the student. The tasks of the lesson must be divided into all types and organizational forms of pedagogical interaction. At the interactive stage of communication (for example, with an online consultation), one or two tasks can be solved, the rest can be transferred to independent study, mutual training, and control.

Distance learning has two options: mastering educational subjects independently using Internet resources and electronic manuals or synchronous and asynchronous interaction between a teacher and students using high technologies, the Internet, various videoconferencing platforms, for example, Zoom, WhatsApp, VKontakte. The second method is the most effective, since, even at a distance, students communicate with teachers, receive from them some instructions, advice on how to complete assignments and sort out mistakes [1,2].

This type of training has positive and negative sides. The positive thing is that people in the age of high technologies are increasingly learning about the world of the Internet, finding the fastest and most effective ways to interact with each other. Being at home, we do not waste our time on the way to the university, but try to direct it to completing various tasks. Additional time is the main advantage of remote learning. The negative side is that a lot of time is spent on distance learning, since in full-time training in pairs, not everyone is being asked, but only a certain part of the group, and in distance learning, you have to send homework to the teacher every time so that he / she notes its completion.

In accordance with order No. 590 / os dated 04/03/2020, the Department of Physical Education and Sports of the RSTU, until 04/10/2020, must develop complexes of independent studies for students with elements of remote control within the discipline "Elective courses in physical culture and sports."

At the meeting of the Department of Physical Education and Sports No. 10 of 04/04/2020, it was decided that the developed by associate professors of the Department of Physical Education and Sports of the RSTU Grigan, S.A., Zenkova, T.A., Lyashko G.I. set of practical tasks for students in the discipline "Elective courses in physical culture and sports" has to be revised, and, moreover, associate professors Grigan S.A., Filippova E.V. and senior teacher Putilina T.A. are to develop a course of online lectures for self-study in the discipline "Elective courses in physical culture and sports" during self-isolation.

Purpose of the study: To offer a set of physical exercises aimed at developing students' strength and allowing, with the help of information technologies, to conduct independent studies during the period of distance learning. Besides, to check its effectiveness during the experiment.

\section{Materials and methods}

The study involved 1-3-year students of the full-time department of the Faculty of Humanities in the amount of 50 people (girls and boys aged 17-20 years).

At the Department of Physical Education and Sports of the RSTU, distance learning was organized in the discipline "Elective courses in physical culture and sports" in the format of independent studies during the isolation of 1-3-year students of the full-time department of the Faculty of Humanities. After analyzing the scientific and methodological literature, generalizing and systematizing the data obtained, a set of exercises was developed for the students to develop strength in the upper limbs girdle. This complex is used at home using available tools. 


\section{Results}

Before the experiment, 1-3-year students of the full-time department of the Faculty of Humanities, when testing strength in the upper limbs girdle, showed, on average, the following results: female students: pulling up from the hang while lying on a low bar -7 reps, flexion and extension of the arms in the lying position - 6 reps. Male students: pullups in the hanging on a high bar - 7 reps, flexion and extension of the arms in the lying position - 9 reps.

The experiment was carried out for three months, from October to January, during the pedagogical experiment, 1-3-year students of the full-time department of the Faculty of Humanities achieved positive changes in these control tests of strength in the upper limbs girdle, and showed, on average, the following results: female students: on a low bar - 12 reps and flexion and extension of the arms in the lying position - 12 reps; male students: pull-up in the hanging on a high bar - 13 reps, flexion and extension of the arms in the lying position - 15 reps. These averages correspond to the silver insignia (GTO programmeReady for Labour and Defence badge).

The numerical values presented in Tables 1 and 2 contain a statistical analysis of the test results of students before and after the experiment.

Table 1. Indicators of preliminary and final testing in the experimental group among female students

\begin{tabular}{|l|c|c|c|}
\hline \multicolumn{1}{|c|}{ Control exercises } & $\begin{array}{c}\text { Before the } \\
\text { experiment } \\
\mathrm{X} \pm \mathrm{m}\end{array}$ & $\begin{array}{c}\text { After the } \\
\text { experiment } \\
\mathrm{X} \pm \mathrm{m}\end{array}$ & $\mathrm{P}$ \\
\hline Pull-up from a hang lying on a low bar & $6.6 \pm 0.1$ & $11.8 \pm 0.1$ & $<0.05$ \\
\hline $\begin{array}{l}\text { Flexion and extension of the arms in the } \\
\text { lying position on the floor }\end{array}$ & $5.2 \pm 0.1$ & $12.0 \pm 0.1$ & $<0.05$ \\
\hline
\end{tabular}

Table 2. Indicators of preliminary and final testing in the experimental group among male students

\begin{tabular}{|l|c|c|c|}
\hline \multicolumn{1}{|c|}{ Control exercises } & $\begin{array}{c}\text { Before the } \\
\text { experiment } \\
\mathrm{X} \pm \mathrm{m}\end{array}$ & $\begin{array}{c}\text { After the } \\
\text { experiment } \\
\mathrm{X} \pm \mathrm{m}\end{array}$ & $\mathrm{P}$ \\
\hline Pull-up from a hang on a high bar & $6.8 \pm 0.1$ & $12.7 \pm 0.1$ & $<0.05$ \\
\hline $\begin{array}{l}\text { Flexion and extension of the arms in the } \\
\text { lying position on the floor }\end{array}$ & $8.2 \pm 0.1$ & $14.8 \pm 0.1$ & $<0.05$ \\
\hline
\end{tabular}

The results presented in the tables indicate that according to the results of the conducted pedagogical experiment, a positive and significant dynamics of all parameters of the development of strength abilities of students engaged in self-learning during the period of distance education was revealed.

To assess the results of the experiment, the dynamics of the results shown in the control tests of strength abilities in the upper limbs girdle was analyzed by comparing the values of the average indicators among students in previous tests and testing after the experiment. The results of the analysis of the dynamics in the control tests among students are given in Tables 1 and 2, which show the average values of the strength abilities of students (X) and their standard deviations $(\mathrm{m})$. Positive and reliable changes in the tests $(\mathrm{P}<0.05)$ allow us to conclude that the program we proposed turned out to be effective for the development of strength in students and allows them to conduct independent studies using information technologies during the period of distance learning.

A beautiful, muscular body has always attracted the attention of the opposite sex. Men also know this fact, so many of them spend huge amount of time in gyms in order to achieve the ideal proportions that women like so much. A particularly massive pilgrimage 
to gyms begins a few months before the start of the summer season. After all, everyone wants to impress girls with their abs or biceps relief.

Although girls do not need to have distinct muscles, some of them still dream about this. When performing exercises on the upper limbs girdle, not only the arms are strengthened, but also the chest area, which is important for women, especially in the summer.

The main muscles of the upper limbs girdle are [3-4]:

1. The biceps are made up of two beams and help to flex the forearm at the elbows. They take on the main load when swinging dumbbells, barbells;

2. The triceps help to extend the forearm at the elbows. They consist of three beams and work when pushing up and spreading arms in an inclined position;

3. The forearms are involved in flexion and extension of the wrists. They get pumped up with any exercise.

To build up the muscle mass of the biceps, one needs to take dumbbells, picking them up according to one's weight (from 1.5 to $4 \mathrm{~kg}$.) Straightening the back in a standing position, one bends their arms with dumbbells (if not possible, one can use water bottles) towards the chest. It is enough to do thirty push-ups in several sets.

The triceps are strengthened like this: we put a chair and, turning our back to it, squat a little and rest our hands on the seat. We gradually lower ourselves, trying to reach the floor with our buttocks, then we rise. We repeat the process ten times in two sets.

Another easy triceps workout is done while sitting on a chair. We arm ourselves with dumbbells, take them in our hands and lift them up above our head. Then we begin to bend our arms, taking them to the side of the head. It is enough to do 15 flexions in several sets, then our triceps are actively worked out. It is important not to forget about the warm-up, otherwise some difficulties may arise: severe muscle pain, rapid fatigue, tendon sprain, etc.

Our job is to alternate between the biceps and triceps. On the first day, we pay attention to the biceps and strengthen them until pain appears. The next day we rest and let the muscles recover. On the third day, we work on strengthening the triceps. On the fourth day we give the tired hands some time to recover. On the fifth day, we repeat the started process, gradually increasing the load $[5,6]$.

Let us consider a set of exercises for those students who do not have dumbbells. Here are the exercises for hands at home in the absence of dumbbells.

One can use the classic push-up in the lying position, the back is straight, the elbows are slightly bent, the hands rest on the floor and are on the chest line slightly wider than the shoulders. Legs rest on the floor and are spread across the width of the hips. Inhaling air, we begin to bend our arms at right angles. The chest does not touch the floor. One should not linger for a long time, it is better to straighten up immediately.

The next technique is similar to the first, with the difference that the thumb and forefinger touch. When pushing up, the ribcage touches the palms. This is how the "narrow grip" training takes place.

There is a wide grip push-up. The initial position is the same as in the first two, only we move our arms as wide as possible. We go down and with the help of the pectoral muscles we rise again. The elbows are bent, the abs are tense, the back is straight.

The fourth technique without dumbbells resembles the classic one. Legs are above the level of the floor, a sofa or chair will do. With these push-ups, most of the load falls directly on the hands.

Push-ups with a clap. Everything is the same as in the classic push-up without dumbbells, only when lifting the torso, we perform a clap. If one can't do such a trick right away, then we take our palms off the floor a little and gradually switch to the clapping. This is a difficult task and is designed for the already strengthened muscles.

All of the above activities go with increasing load. We start with the first task, work it through for 1 week and move on to the next. Each task takes 1 week. When severe muscle 
pain appears, we restrict movement to avoid injury.

Exercises for men.

If there is no barbell or dumbbells at home, training without the use of equipment is a very good opportunity, since they do not require any costs and can be carried out anywhere, but only comfortable clothes and a couple of meters of free space are required.

When exercising at home, one needs to adhere to the following rules $[7,8]$ :

1. Workouts should be done in a room or outdoors;

2. When exercising, the air must circulate well;

3. It is recommended to train at a temperature of $23^{\circ} \mathrm{C}$;

4. It is advised to warm up the muscles beforehand;

5. One should stretch his or her muscles for a couple of minutes after exercising.;

6. During the lesson, one should not feel thirsty; for this, drinking water needs to be prepared in advance (0.5-1 1.).

Start your workout with squats, 2-3 sets of 20-25 reps. For a greater load, you can reduce the speed of execution: 15 seconds in a squatting position and 15 seconds in an erect position. If the load is still light, then do the exercise on one leg, holding the chair with your hand.

Followed by: 3-4 sets of 30-40 seconds. For this exercise, you need to stand on your hands near the wall. And then stand on your feet and transfer the weight on your toes: 2-3 sets of 15-20 reps.

Pull-ups: 3-4 sets of 8-12 reps.

Push-ups: 3 sets of 30-40 reps. Arms along the body: 2 sets of 8-10 reps. Crunches: 2 sets.

Advice: do not forget to train the thoracic region, a few simple exercises will help you with this: stand up, leaning against the wall with four points (heels, buttocks, shoulder blades, back of the head). Fold the palms in front of you and slowly lift up, without bending at the elbows, so that the inner surface of the hands is pressed against the ears. Try to gently and softly touch the wall with your thumbs, without lifting your body from the wall and not bending your elbows. Fix the position for a minute, and slowly relax, letting your hands down.

The next exercise: lie on your chest, arms bent at the elbows along the body. Touch the floor with your forehead. The palms are firmly in contact with the floor so that the abducted thumbs touch the shoulders. Press your hips to the floor. With the help of your hands, squeeze the torso from the floor, bending your back backwards as far as possible, throw your head back. The hips and lower abdomen do not come off the floor. Having straightened to the maximum, try to lift your hands off the floor, thereby depriving yourself of support. Hold this position for thirty to forty seconds. Then return to the starting position. The exercise should be repeated once.

Next exercise: lie down again, arms at the side of the body. Bend your elbows, touch your temples with your palms, spread your elbows to the sides as much as possible and bend the body, rising higher to linger for thirty to forty seconds. And return to the starting position. 12-17 reps, hold 3-5 seconds.

Exercises for women.

For women, you can use a gentle push-up technique. We do push-ups on bent knees, keeping the torso in a straight position. The gaze is directed forward. No sagging belly or raised buttocks.

There are three ways to do it: arms spaced shoulder-width apart; hands at the same level, but the hands are directed towards each other; arms shoulder-width apart, but the hands are directed straight in front of you, parallel; arms shoulder-width apart are directed in opposite directions. By doing these simple movements, you use all types of muscles. Little by little we move to straightened legs, that is, we do not kneel. 
Training program for men and women [9]:

It is recommended to pump up the arms each second day, allowing the muscles to recover:

1. One day we work out biceps, the other day - triceps for a uniform load;

2. Exercise to the limit (muscle soreness) for best results;

3. If it is not possible to train after each second day, then two days off will do. We pump up biceps and triceps alternately in one day.

Let's name the advantages of self-isolation:

1. Self-training. You can spend more time on what you need, you can skip unnecessary, in your opinion, items, you can manage your time as you want.

2. Comfortable environment. With distance learning, the student can study where he likes.

3. Relationships in a group cease to be a problem - even if a student does not get along with classmates, it makes almost no difference in distance learning, shy students who are shy of their physique, having gotten rid of the group pressure, show the best results in distance learning.

4. Own pace. You study at home, at a time convenient for you. No need to waste time on the way to school and back. The student does not need to adapt to anyone, he can master the material at a speed suitable for himself or herself.

5. Available teaching materials. There are a lot of free electronic tutorials on the net. Students and teachers are not limited to the assortment of the student library.

\section{Discussion}

The experience of the widespread introduction of distance learning due to the pandemic shows that further pedagogical research in this area is required, aimed at improving the quality of education. At the moment, in many educational institutions, students are not receiving training at the same level as before the pandemic, due to a number of factors related not only to technical support, but also to the unpreparedness of participants in the educational process to switch to distance learning. The existing problems indicate the need to improve the quality of distance learning, use the full range of opportunities provided by modern means of Internet technologies, as well as prepare students and teachers to work in a remote format. With an insufficient level of motivation, both students and teachers may form a negative attitude towards the use of Internet technologies in the educational process, which will lead to a significant decrease in the quality of education [10-12].

The organization of pedagogical interaction on the basis of a competent approach to education makes it possible to orient the goal-setting of classes towards the formation and development of the student's skills and abilities. Integration of the learning and social environment will allow to influence changes at the level of the student's personality. The creation of communities and the consultative nature of communication solve the student's personal problems, allow him or her to assert himself or herself and provide pedagogical support [13-15].

The complex use of modern technologies is necessary, including educational, managerial, communicative and informational technologies.

To eliminate the demotivators of distance learning, it is necessary to diversify the forms of communication, the time of their conduct, and geographic accessibility.

It is necessary to follow the rules in physical education classes in a distance mode:

- age-specific and individual physical and functional characteristics of trainees;

- a gradual increase in physical activity during the distance learning period;

- regularity in physical exercise;

- a variety of forms of motivation to engage in physical exercises and control over various aspects of their implementation. 


\section{Conclusions}

The experiment made it possible to reveal that at home, using modern technologies, it is possible to achieve positive results in these control tests, and show, on average, the following results: female students: pulling up from the hang while lying on a low bar - 12 reps, flexion and extension of the arms in the lying position - 12 reps. Male students: pullups in the hanging on a high bar - 13 reps, flexion and extension of the arms in the lying position - 15 reps. These averages correspond to the silver insignia (GTO programmeReady for Labour and Defence badge).

The numerical values presented in Tables 1 and 2 contain a statistical analysis of the test results of students before and after the experiment. Positive and reliable changes in the tests $(\mathrm{P}<0.05)$ allow us to conclude that the program we proposed turned out to be effective for the development of strength in students and allows them to conduct independent studies using information technologies during the period of distance learning. This is evidenced by the results in tests such as female students: pull-ups from the hang while lying on a low bar, flexion and extension of the arms in the lying position; students: pull-up in the hanging on a high bar, flexion and extension of the arms in the lying position.

\section{References}

1. Choice of the people 5, (2020). URL: http: //vybor-naroda.org/lentanovostey/165427prepodavateli-vuzov-protiv-distancionnogo-obrazovanija.html. (date of access: 1.10.2020).

2. V.A. Baronenko, L.A. Rapoport, Health and physical culture of a student: Textbook (Alfa, Moscow, 2017).

3. A.A. Bishaeva, Physical culture: Textbook (Academy, Moscow, 2018).

4. S.A.Grigan, A.M. Grigan, Collection of scientific papers Sworld 4 (44), 20-24 (2016).

5. V.Ya. Kikotia, I.S. Barchukova, Physical culture and physical training: Textbook (Unity, Moscow, 2017).

6. R.C. Chick, G.T. Clifton, K.M. Peace, B.W. Propper, D.F. Hale, A.A. Alseidi, T.J. Vreeland, J Surg Educ 77 (4), 729-32 (2020).

7. D. Shah, Climacteric 19 (1), 3-6 (2016).

8. E.T.H. Norman, D. Huegel, Front Digit Humanit 3, 5 (2016).

9. S. Kaup, R. Jain, S. Shivalli, S. Pandey, S. Kaup, Indian J Ophthalmol 68 (6), 1220-1 (2020).

10. S. Billett, Stud EducAdults 34 (1), 56-67 (2016).

11. R.M. Harden, J.M. Laidlaw, Med Teach 35 (1), 27-31 (2013).

12. T. Brockfeld, B. Müller, J. de Laffolie, Med Educ Online 23 (1), 1555434 (2018).

13. E. Molloy, R. Ajjawi, M. Bearman, C. Noble, J. Rudland, A. Ryan, Med Educ. 54 (1), 33-9 (2020).

14. D. Memmert, L. Almond, D. Bunker, J. Butler, F. Fasold, L. Griffin, S. Nopp, Research Quarterly for Exercise and Sport 86 (4), 347-359 (2015). 